\title{
Low-Dose Lung Radiation Therapy for COVID-19 Lung Disease: A Preclinical Efficacy Study in a Bleomycin Model of Pneumonitis
}

\author{
Mark R. Jackson, DPhil, * Katrina Stevenson, MSc, * \\ Sandeep K. Chahal, MRes, ${ }^{*}$ Emer Curley, MSc, ${ }^{\dagger}$ George E. Finney, MSc, \\ Rodrigo Gutierrez-Quintana, MVM, ${ }^{*}$, Evarest Onwubiko, MSc, \\ Angie Rupp, DipECVP, ${ }^{\S}$ Karen Strathdee, BSc, ${ }^{*}$ Karin Williams, PhD,* \\ Megan K.L. MacLeod, PhD, Charles McSharry, PhD, and \\ Anthony J. Chalmers, MD, PhD*
}

\author{
${ }^{*}$ Institute of Cancer Sciences, University of Glasgow, United Kingdom; ${ }^{\dagger}$ Cancer Research UK Beatson \\ Institute, Glasgow, United Kingdom; ${ }^{\ddagger}$ Institute of Infection, Immunity and Inflammation, University \\ of Glasgow, United Kingdom; and ${ }^{\S}$ School of Veterinary Medicine, University of Glasgow, United \\ Kingdom
}

Received Mar 10, 2021; Revised Aug 5, 2021; Accepted for publication Aug 24, 2021

Purpose: Low-dose whole lung radiation therapy (LDLR) has been proposed as a treatment for patients with acute respiratory distress syndrome associated with SARS-CoV-2 infection, and clinical trials are underway. There is an urgent need for preclinical evidence to justify this approach and inform dose, scheduling, and mechanisms of action.

Methods and Materials: Female C57BL/6 mice were treated with intranasal bleomycin sulfate ( 7.5 or 11.25 units $/ \mathrm{kg}$, day 0$)$ and then exposed to whole lung radiation therapy $(0.5,1.0$, or $1.5 \mathrm{~Gy}$, or sham; day 3$)$. Bodyweight was measured daily, and lung tissue was harvested for histology and flow cytometry on day 10. Computed tomography lung imaging was performed before radiation (day 3 ) and pre-endpoint (day 10).

Results: Bleomycin caused pneumonitis of variable severity, which correlated with weight loss. LDLR at 1.0 Gy was associated with a significant increase in the proportion of mice recovering to $98 \%$ of initial bodyweight, and a proportion of these mice exhibited less severe histopathologic lung changes. Mice experiencing moderate initial weight loss were more likely to respond to LDLR than those experiencing severe initial weight loss. In addition, LDLR (1.0 Gy) significantly reduced bleomycin-induced increases in interstitial macrophages, CD103+ dendritic cells (DCs), and

Corresponding author: Anthony J. Chalmers, MD, PhD; E-mail: anthony.chalmers@glasgow.ac.uk

This study was made possible by the following funding sources: A.C.: Medical Research Council Research Grant (MRR0094731). A.C., K.S.: Cancer Research UK Radiation Research Centre of Excellence (C16583/ A28803). K.S. Cancer Research UK Clinician Scientist Fellowship (Ross Carruthers, C52808/A23920). M.K.L.M.: Wellcome Trust [210703/Z/18/ Z]. C.M.: BPF Medical Research Trust (SCO1318).

Disclosures: none

Data sharing statement: Research data are stored in an institutional repository and will be shared upon request to the corresponding author.
Supplementary material associated with this article can be found, in the online version, at doi:10.1016/j.ijrobp.2021.08.029.

Acknowledgments-The authors gratefully acknowledge the following for their contributions to this research: University of Glasgow Flow Core Facility; Colin Nixon (CRUK Beatson Institute Core Histology Service); David Lewis (CRUK Beatson Institute) for CT quantification advice; Lesley Gilmour (University of Glasgow) for in vivo experimental design advice; Karen Blyth (CRUK Beatson Institute); and Ashley Tatum for project management advice. 
neutrophil-DC hybrids. Overall, bleomycin-treated mice exhibited significantly higher percentages of nonaerated lung in left than right lungs, and LDLR (1.0 Gy) limited further reductions in aerated lung volume in right but not left lungs. LDLR at 0.5 and $1.5 \mathrm{~Gy}$ did not improve bodyweight, flow cytometric, or radiologic readouts of bleomycininduced pneumonitis.

Conclusions: Our data support the concept that LDLR can ameliorate acute inflammatory lung injury, identify 1.0 Gy as the most effective dose, and provide evidence that it is more effective in the context of moderate than severe pneumonitis. Mechanistically, LDLR at 1.0 Gy significantly suppressed bleomycin-induced accumulation of pulmonary interstitial macrophages, CD103+ DCs, and neutrophil-DC hybrids. ( 2021 The Authors. Published by Elsevier Inc. This is an open access article under the CC BY license (http://creativecommons.org/licenses/by/4.0/)

\section{Introduction}

To date (August 2021), infection with SARS-CoV-2 has been associated with more than 4,250,000 deaths globally. ${ }^{1}$ Infection with SARS-CoV-2 can cause a syndrome termed COVID-19, the severity of which varies widely. A small proportion of patients develop severe pneumonia and acute respiratory distress syndrome ${ }^{2}$ characterized by pathologic inflammation, the severity of which correlates with mortality. ${ }^{3}$ The histopathologic features and systemic complications are characteristic of acute respiratory distress syndrome and cytokine release syndrome. ${ }^{4}$ Pathologic features include infiltration of alveoli by macrophages, lymphocytes, and neutrophils and high levels of proinflammatory cytokines such as interleukins- 6 and $1 b$, interferon gamma, and tumor necrosis factor. ${ }^{5,6}$

There are very few effective treatments for COVID-19 lung disease. The RECOVERY trial (United Kingdom) showed that dexamethasone treatment was associated with a reduction in 28-day mortality compared with standard of care among hospitalized patients who required ventilatory support (relative risk, 0.65) or oxygenation (relative risk, $0.80){ }^{7}$ More recently, the REMAP-CAP trial reported improvements in survival and time to recovery after dual therapy with tocilizumab and sarilumab (unpublished data), and the anti-inflammatory agent colchicine has been reported to reduce hospitalisation and mortality in patients with COVID-19 with at least 1 risk factor for complications. ${ }^{8}$ Although some of these studies await peer review, the early clinical data support the concept that acute inflammation is the key pathologic process in COVID-19 lung disease and indicate that broad spectrum immunosuppressive therapies may be of therapeutic value.

Low-dose whole lung radiation therapy (LDLR; radiation doses $\leq 1.5 \mathrm{~Gy}$ ) was used extensively as a treatment for pneumonias of various etiologies in the preantibiotic era. ${ }^{9}$ In various preclinical models of inflammation, LDLR has been shown to induce anti-inflammatory cytokine production, reduce leukocyte-endothelial adhesion, and repolarize myeloid and lymphoid cells toward immune-suppressive phenotypes. ${ }^{10}$ Recent preclinical studies have generated preliminary data to indicate that LDLR (0.5-1.0 Gy) reduces pneumonitis in lipopolysaccharide and influenza virus mouse models. ${ }^{11}$ These diverse but limited bodies of evidence have underpinned early phase clinical trials of LDLR for acutely unwell patients with COVID-19 in several countries, including the United States, India, and Spain. The safety of the intervention is supported by preliminary results from phase 1 trials, ${ }^{12,13}$ in which early signals of efficacy were reported, including in elderly patients with severe COVID-19 lung disease, most of whom had multiple comorbidities. ${ }^{12}$ Very recently, however, the first randomized clinical data to be published $(n=22)$ showed no benefit of 1.0 Gy whole lung radiation therapy over sham irradiation in ventilation-dependent patients with severe COVID19 pneumonia. $^{14}$

This therapeutic approach has generated intense controversy. ${ }^{15-18}$ Although expressing diverse opinions, the vast majority of stakeholders have emphasized the urgent need for high-quality preclinical data to (1) justify (or not) the commencement of clinical studies, (2) elucidate mechanisms of efficacy, and (3) inform decisions on radiation dose, scheduling, and target volume. ${ }^{19}$ To address this need, we undertook preclinical studies using a mouse model of bleomycin-induced pneumonitis. Many pathophysiologic changes of COVID-19 lung disease (epithelial cytopathy, endotheliitis, inflammatory infiltrates, surfactant loss) are reproduced in pneumonitis induced by inhaled bleomycin. ${ }^{20,21}$ Indeed, single cell sequencing studies of mouse bleomycin ${ }^{22}$ and COVID-19 ${ }^{23,24}$ pneumonitis have shown pathogenic SPP1 ${ }^{\text {pos }}$ macrophage orthologues expressing key inflammatory mediators to be prominent in both conditions, along with profoundly reduced expression of anticoagulant and antiapoptotic protein $\mathrm{S}$ in alveolar macrophages. Exogenous protein $\mathrm{S}$ is protective of bleomycin pneumonitis ${ }^{25}$ and has been proposed as a potential treatment for COVID-19. ${ }^{26}$

To test the hypothesis that LDLR would reduce the severity of bleomycin-induced acute lung injury by exerting suppressive effects on cellular and molecular components of the inflammatory response, we measured the effects of $0.5,1.0$, and 1.5 Gy whole lung irradiation on bodyweight (primary endpoint), pulmonary cytology and histology, and lung computed tomography (CT) appearances (secondary endpoints). Our data show that 1.0 Gy LDLR enhances recovery in a proportion of bleomycin-treated mice, with corresponding improvements in lung histopathology and imaging parameters and modulation of specific immune 
cell populations. Effects were more marked in mice with moderate rather than severe pneumonitis.

\section{Methods and Materials}

\section{Reagents}

All reagents were purchased from Biolegend unless otherwise stated. Bleomycin sulfate was obtained from European Pharmacopeia EDQM, Council of Europe, France. Intranasal bleomycin dose was 11.25 units/kg except in the initial pilot study, when 7.5 units $/ \mathrm{kg}$ was also used.

\section{Experimental pneumonitis}

Bleomycin generates a well-established murine model of pneumonitis $^{21}$ with a dynamic pathology similar to that of COVID-19. Female, 11- to 13-week-old C57BL/6 mice (Charles River Laboratories) were administered 1 intranasal $40 \mathrm{~mL}$ dose of bleomycin sulfate (7.5 or 11.25 units $/ \mathrm{kg}$ ) or phosphate-buffered saline (PBS) vehicle control under light isoflurane anesthesia. Mice were maintained in a pathogenfree facility, provided with additional high calorie, soft diet to ease feeding, and monitored daily for wellbeing and change in bodyweight. Those demonstrating signs of illness such as lethargy, isolation, reduced mobility, altered respiration, or $\geq 25 \%$ weight loss were humanely euthanized. The experimental design optimized mice numbers to comply with the principles of replacement, reduction, and refinement for humane animal research. Procedures were governed by the Animals Scientific Procedures Act 1986 and approved by home office license PP6245051.

\section{Low-dose lung radiation therapy}

Bleomycin-treated mice exhibiting a day 3 relative bodyweight area under the-curve (AUC) $\leq 2.92$ were randomized to receive LDLR or sham irradiation. Anaesthetized mice were irradiated with $\operatorname{LDLR}(0.5,1.0$, or $1.5 \mathrm{~Gy}$ or sham) on day 3, using the Small Animal Radiation Research Platform (SARRP) developed by XStrahl. A $220 \mathrm{kVp}, 13 \mathrm{~mA}$ x-ray beam was used with a dose rate of approximately $280 \mathrm{cGy} /$ min at the chosen aperture size. Treatment was delivered with anterior and posterior parallel opposed fields. The broad focal spot $(5.5 \mathrm{~mm})$ was used, and the SARRP's motorized variable collimator set to an aperture size of $20 \times 20 \mathrm{~mm}$ to ensure full coverage of both lungs. Mice were euthanized on day 10, and lung tissue was harvested for experimental endpoint analysis as described in the following sections.

\section{Computer tomography assessment of lung changes}

Lung changes were measured using the SARRP's in-built cone beam CT (CBCT) function to image anaesthetized mice on days 3 (preirradiation) and 10 (experimental endpoint). Images were reconstructed using the Feldkamp, Davis, and Kress CBCT reconstruction algorithm from 1440 projections taken at $60 \mathrm{kVp}$ and $0.8 \mathrm{~mA}$ using the fine focal spot $(1 \mathrm{~mm})$. The absorbed dose associated with each CBCT was calculated to be approximately $4.8 \mathrm{cGy}$. For quantification of aerated lung volumes, Hounsfield unit (HU) clinical ranges were used: poorly aerated lung was defined as -500 to $-100 \mathrm{HU}$ and normo-aerated was defined as -900 to $-500 \mathrm{HU}$. Images were analyzed using the Lung CT analyzer module from the 3D Slicer software extension SlicerCIP. ${ }^{27,28}$ Additional detail is provided in Supplementary Information (Lung CT quantification).

\section{Tissue collection}

Mice were euthanized by terminal intraperitoneal injection of $100 \mathrm{~mL}$ sodium pentobarbital $(200 \mathrm{mg} / \mathrm{mL})$ and cardiac exsanguination. The trachea was exposed, a small transverse opening was cut between cartilage rings, and a ligature was tied loosely distal to the cut. The protruding 0.5 $\mathrm{cm}$ tip of a cannula sheath around a $23 \mathrm{G}$ syringe needle was inserted into the opening, and the ligature was tightened. The lungs were lavaged twice with $0.8 \mathrm{~mL}$ PBS and then perfused via the right ventricle with cold PBS until they blanched, after which lungs and heart were removed en bloc. The left lobe of the lung was excised, submerged in $4 \%$ neutral buffered formalin fixative for 24 hours, and processed for histology, and the right lung lobes were processed for cytology.

\section{Lung histology}

Serial $4 \mu \mathrm{m}$ sections of the left lobe were cut and stained with hematoxylin and eosin and Masson's trichrome and evaluated independently by a veterinary pathologist and a pulmonary immunologist, both of whom were blinded to the experimental treatment. In brief, semiquantitative scoring (described in detail in supplementary information) examined the extent of interstitial mononuclear cell infiltrates, specifically interstitial (to intra-alveolar) macrophage infiltrates and perivascular/peribronchiolar lymphocyte aggregates.

\section{Lung tissue cytology}

Small pieces of right lung were incubated with dispase (3.2 $\mathrm{mg} / \mathrm{mL}$; Roche), collagenase P $(0.4 \mathrm{mg} / \mathrm{mL}$; Roche), and DNAse I $\left(0.2 \mathrm{mg} / \mathrm{mL}\right.$; Sigma) in $2-\mathrm{mL}$ RPMI at $37^{\circ} \mathrm{C}$ in a shaking incubator for 40 minutes. Lung pieces were transferred into a $100 \mathrm{~mm}$ strainer, and a single cell suspension was prepared and filter rinsed to transfer all cells to a 50$\mathrm{mL}$ tube. Red blood cells were lysed by RBC lysis buffer (ThermoFisher) and viable cells were counted. Next, cells were Fc blocked with 24G2 antibody and normal mouse serum for 10 minutes and then incubated with fluorescently 
A
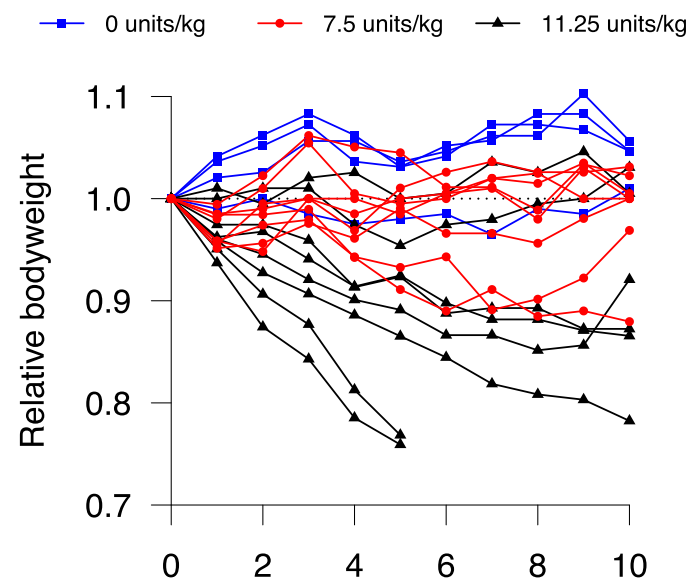

Time (days)

C

Day 3

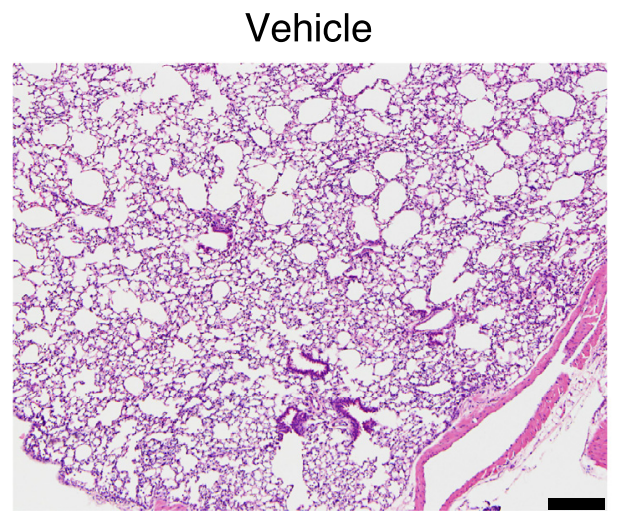

$\mathrm{D}$

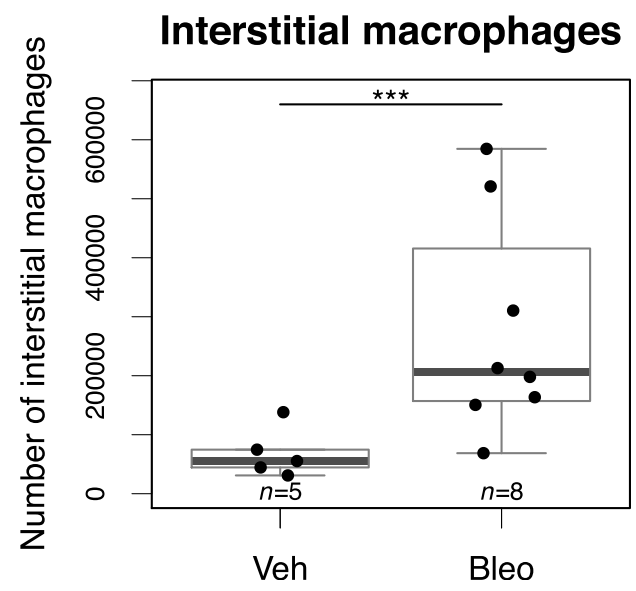

B

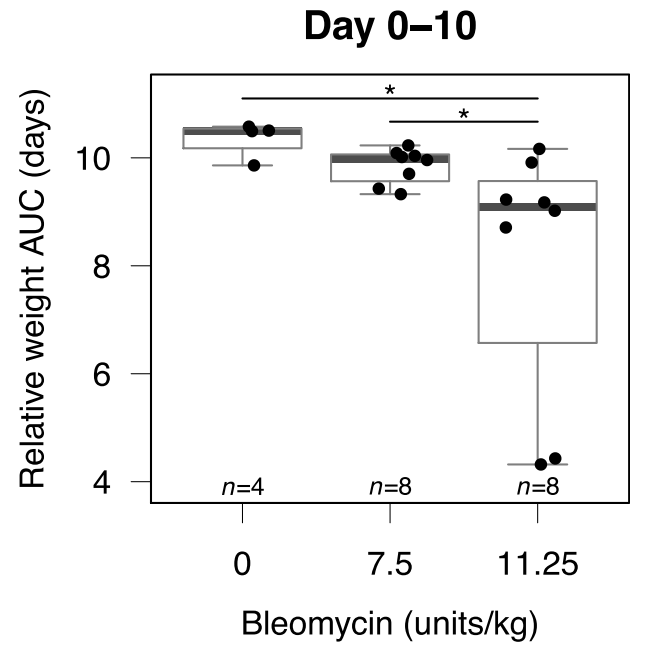

Bleomycin

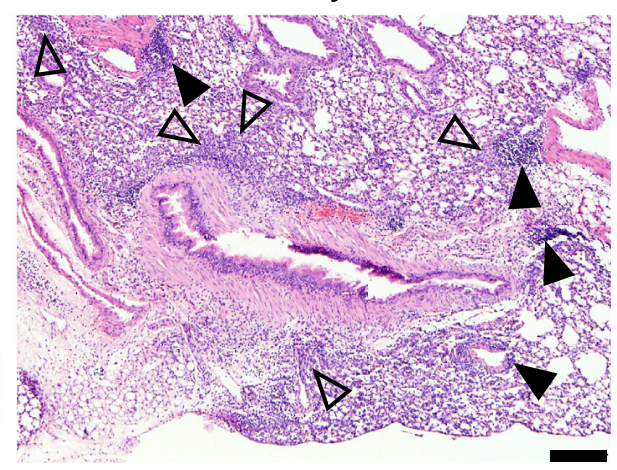

Alveolar macrophages

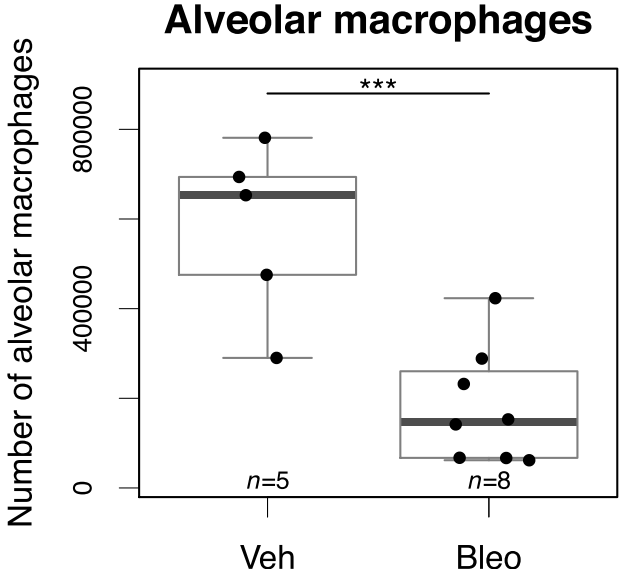

Fig. 1. Bleomycin induces a variable pneumonitis in young adult, female C57BL/6 mice. (A) Relative mouse bodyweight after intranasal administration of bleomycin. (B) Area under the curve (AUC) of relative mouse bodyweight up to day 10. (C) Pulmonary histology 3 days after treatment with vehicle (left) and bleomycin (11.25 units/kg, right). Macrophage infiltrates are annotated with open arrows and lymphocyte aggregates with filled arrows. Hematoxylin and eosin stain, scale bars: $200 \mathrm{~mm}$. (D) Flow cytometric analysis of macrophages in mouse lung at day 3 after bleomycin treatment (11.25 units $/ \mathrm{kg}$ ). (E) Mice with day 3 relative bodyweight AUC $£ 2.92$ were selected for inclusion in efficacy studies. The corresponding relative bodyweights of excluded and included (randomized) mice are presented as an example. (F) Bodyweights of control mice treated with intranasal phosphate-buffered saline (PBS) and subjected to $1.0 \mathrm{~Gy}$ whole lung irradiation 24 hours later. Groups compared by Kruskal-Wallis and post hoc Wilcoxon pairwise test, $* P<.05$, $* * * P<.001$. 
$E$

Thresholding

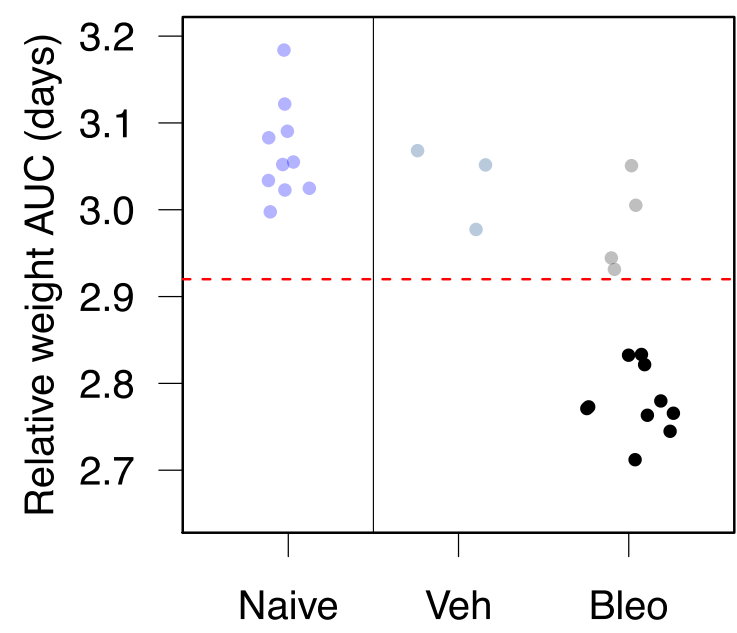

Naive Veh Bleo

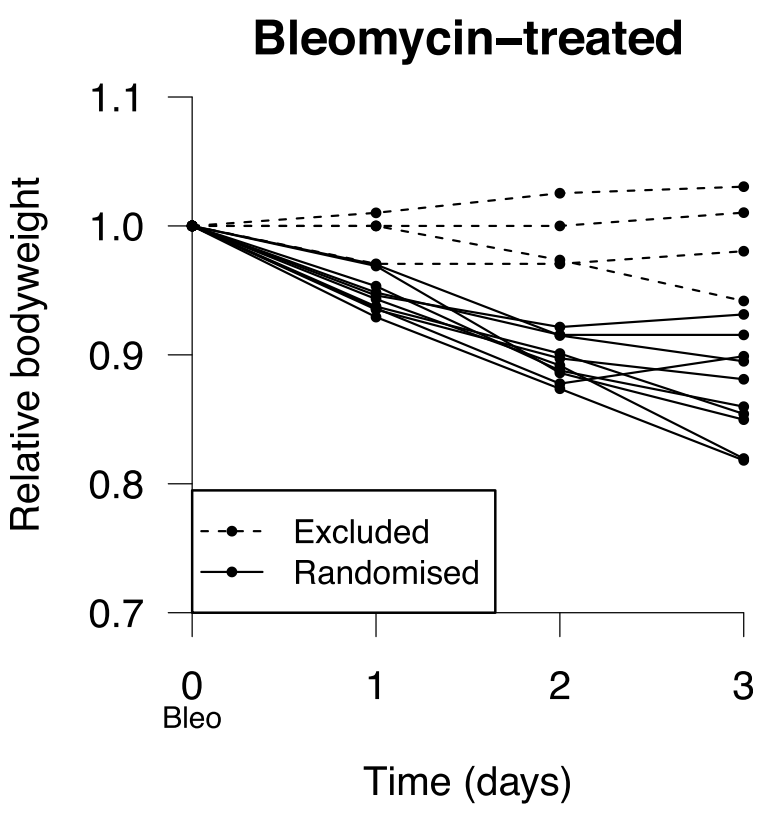

F

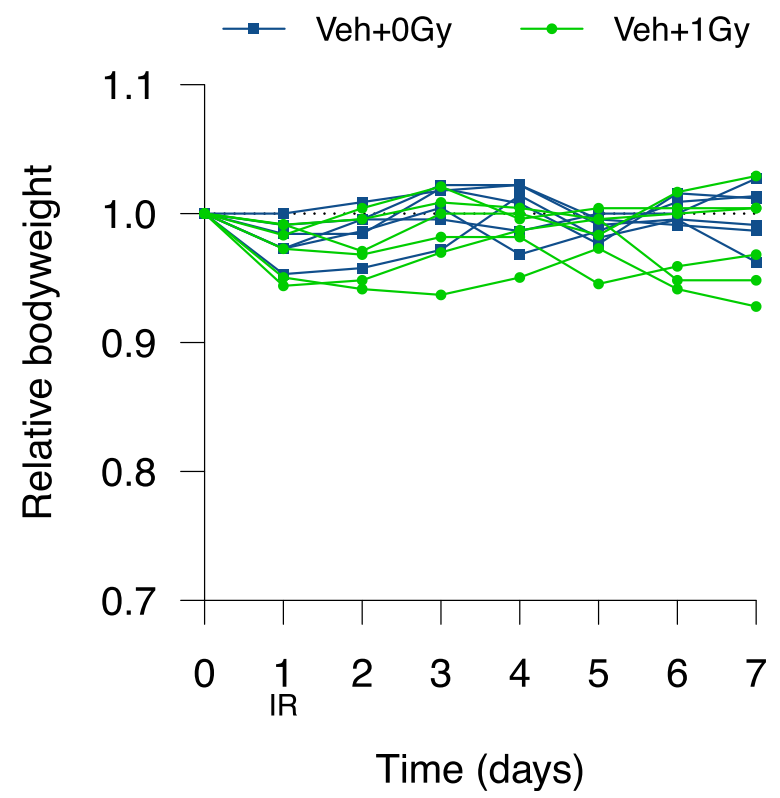

Fig. 1. Continued.

labeled antibodies for 20 minutes at $4{ }^{\circ} \mathrm{C}$. Antibodies are listed in Table E1.

After PBS washing, cells were incubated with viability dye (Zombie Aqua) for 20 minutes at $4^{\circ} \mathrm{C}$, washed with FACS buffer (PBS with 2\% FCS, $2 \mathrm{mM}$ EDTA, and $5 \mathrm{mM}$ sodium azide), fixed with $2 \%$ paraformaldehyde for 20 minutes at $4^{\circ} \mathrm{C}$, washed again with FACS buffer, and then stored at $4^{\circ} \mathrm{C}$. Flow cytometry data were acquired on a BD Fortessa and analyzed using FlowJo (version10, BD Biosciences). The gating strategy ${ }^{29}$ is shown in Figure E1.

\section{Statistical analysis}

Statistical analyses were performed in R $3.6 .3^{30}$ using the packages "MESS," 31 "survival," ${ }^{32}$ and "survminer." ${ }^{33}$ Box and whiskers were plotted according to the Tukey method. Statistical tests used and group sizes are indicated for each experiment. Additional detail on use of AUC and recovery probability analyses is presented as supplementary information. 


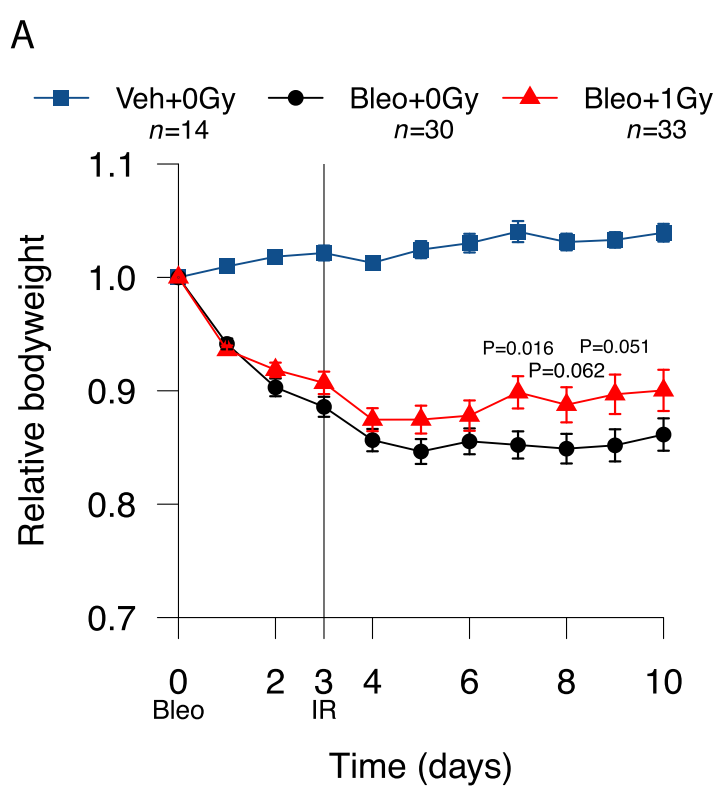

Fig. 2. Low-dose whole lung radiation therapy (LDLR) promotes recovery of bodyweight in a subset of bleomycin-treated mice. (A) Relative bodyweight (mean \pm standard error of the mean [SEM]) of bleomycin-treated mice treated with LDLR (1.0 Gy) or sham irradiation on day 3. Day-by-day comparison of sham and irradiated groups performed by $t$ test. (B) Relative bodyweight of individual mice; recovery defined as a return to $96 \%, 98 \%$, or $100 \%$ of baseline bodyweight (green boxes) after day 3. (C) KaplanMeier analysis of recovery to $98 \%$ of baseline bodyweight; groups compared by log-rank test. (D) Kaplan-Meier analysis of mouse survival. Mice exhibiting severe weight loss were culled to comply with humane endpoint. Groups compared by log-rank test. (E) Relative bodyweight area under the curve (AUC) values up to day 3 (before LDLR), grouped according to eventual study outcome. Treatment groups compared by $t$ test and subgroups compared by 1-way analysis of variance (ANOVA) with post hoc Tukey test. Mice whose bodyweight returned to $98 \%$ of baseline were classified as recovered. Mice euthanized early owing to an excessive reduction in bodyweight were classified as having experienced severe weight loss. ${ }^{*} P<.01,{ }^{* * *} P<.001$.

\section{Results}

Initially, pilot studies were conducted to characterize the bleomycin-induced pneumonitis model and establish optimum dosing and scheduling parameters. Using mouse bodyweight as a marker of systemic response, we observed variable responses to intranasal bleomycin (Fig. 1A), as reported in other studies. AUC analysis revealed that, despite measurable weight loss at day 3 (Fig. E2), by day 10 the bodyweight of mice treated with 7.5 units $/ \mathrm{kg}$ bleomycin was not significantly different from controls (Fig. 1B). In contrast, administration of 11.25 units $/ \mathrm{kg}$ induced progressive weight loss in the majority of mice, with $25 \%$ exhibiting a severe reduction that triggered humane endpoint euthanization but $25 \%$ failing to show a demonstrable response (Fig. 1A,B). Histologic assessment

\section{B}

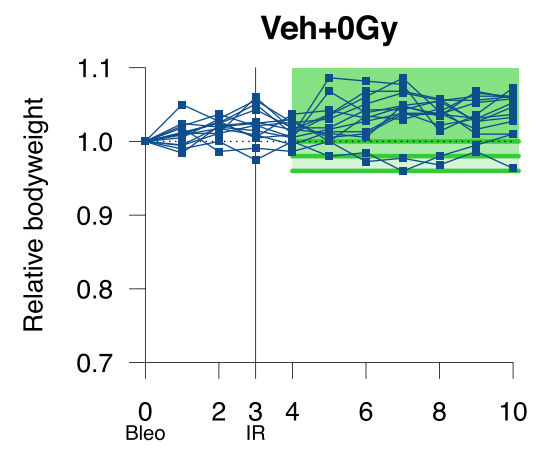

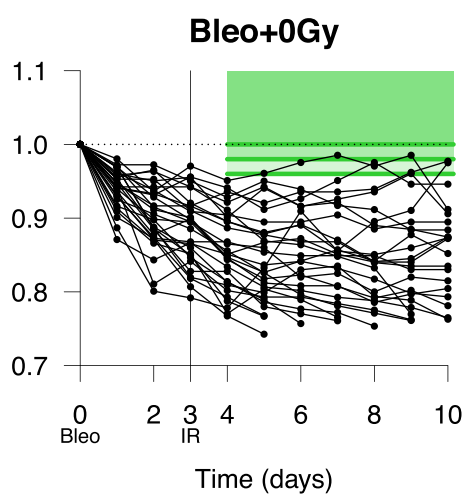

Fig. 2. Continued.

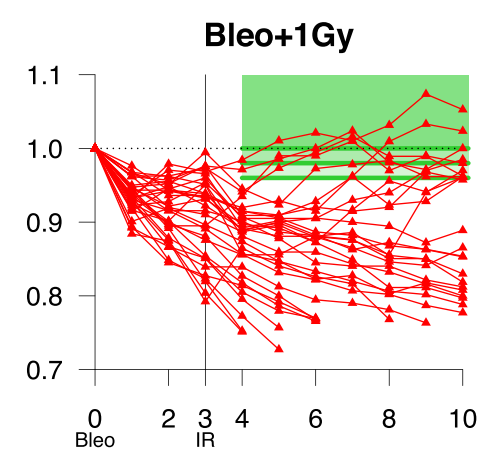




\section{C}
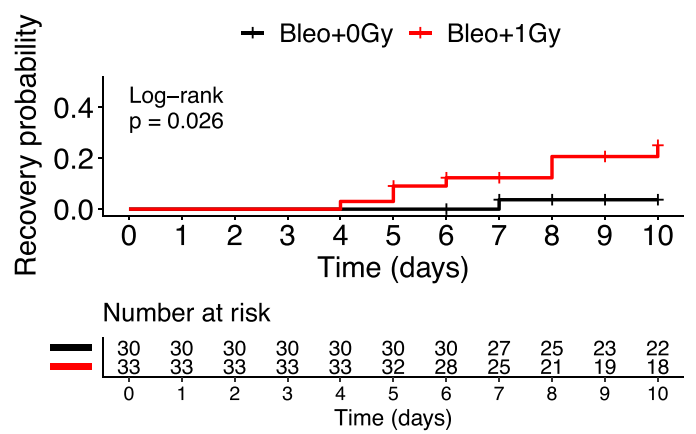

Cumulative number of events

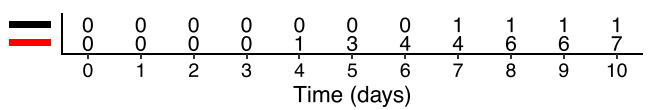

D
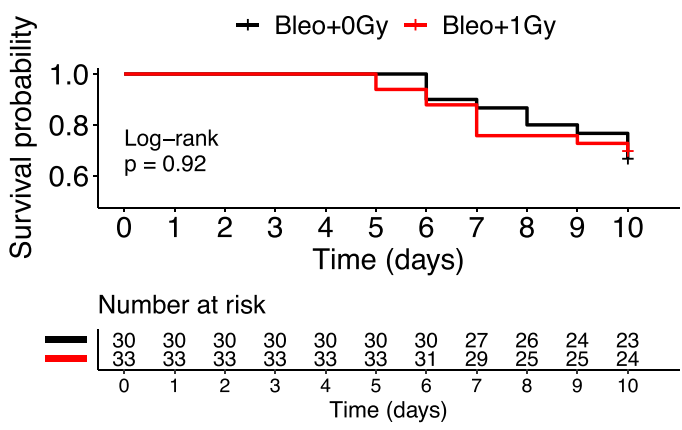

Cumulative number of events

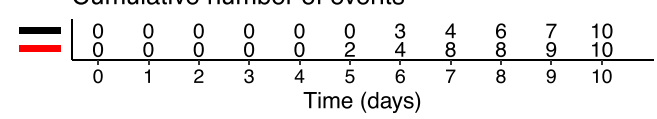

E

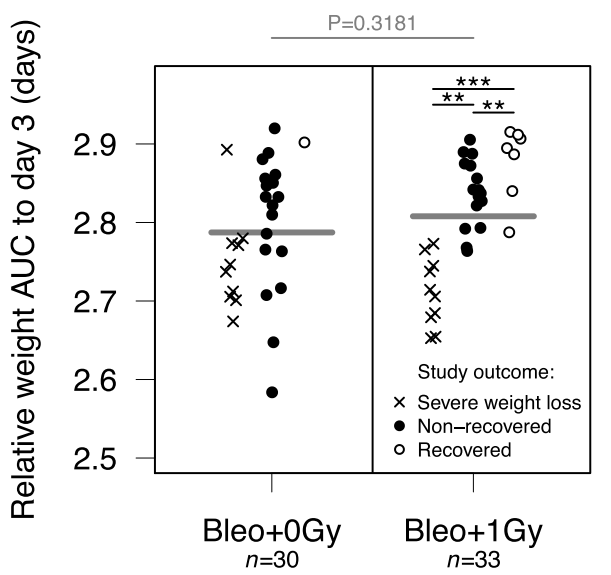

Fig. 2. Continued.

on day 3 revealed multifocal, small, interstitial to intraalveolar macrophage infiltrates and appreciable but small perivascular and peribronchiolar lymphocyte aggregates in the majority of mice (Fig. 1C). These were accompanied by a robust reduction in alveolar macrophages and an increase in interstitial macrophages measured by flow cytometry in dispersed lung tissue (Fig. 1D).

To mirror the clinical scenario in which LDLR would only be considered in patients exhibiting moderate to severe COVID-19 lung disease, we opted to deliver LDLR 3 days after bleomycin treatment. Mice showing minimal weight loss at day 3 were excluded and those exhibiting a sustained drop in bodyweight (defined by day 3 AUC $\leq 2.92$ ) were randomly allocated to receive LDLR or sham irradiation (Fig. 1E). Treatment of vehicle-only control mice with LDLR (1.0 Gy) was well tolerated with no effect on bodyweight and no detectable deviation from normal behavior (Fig. 1F).

Despite the variability inherent to the bleomycin model, treatment with $1.0 \mathrm{~Gy}$ was associated with a modest increase in mean bodyweight in irradiated versus sham- irradiated mice from day 5 onward (Fig. 2A). Bodyweight plots for individual mice (Fig. 2B) illustrate the variable response to bleomycin and identify a subpopulation of irradiated mice recovering to at least $96 \%$ of baseline bodyweight. Kaplan-Meier analysis demonstrated a statistically significant increase in the proportion of irradiated mice recovering to at least $98 \%$ of initial bodyweight after day 3 $(21.2 \%, \mathrm{n}=33)$ compared with sham-irradiated mice $(3.3 \%, \mathrm{n}=30 ; P=.0265)$, with recovery also occurring earlier (Fig. 2C). This definition of recovery (regaining 98\% of initial weight) was used as a reference in subsequent analyses. Recovery was also significantly increased in irradiated mice if a recovery threshold of $100 \%$ was imposed $(P=.0230)$, and a strong trend was observed at $96 \%$ $(P=.0776)$ (Table E2). Of note, treatment with LDLR did not increase the likelihood of an adverse outcome, with no difference in the proportions of irradiated and sham-irradiated mice experiencing severe weight loss (Fig. 2D, Fig. E3).

To identify biomarkers predicting efficacy, we looked for correlations between the severity of the bleomycin 


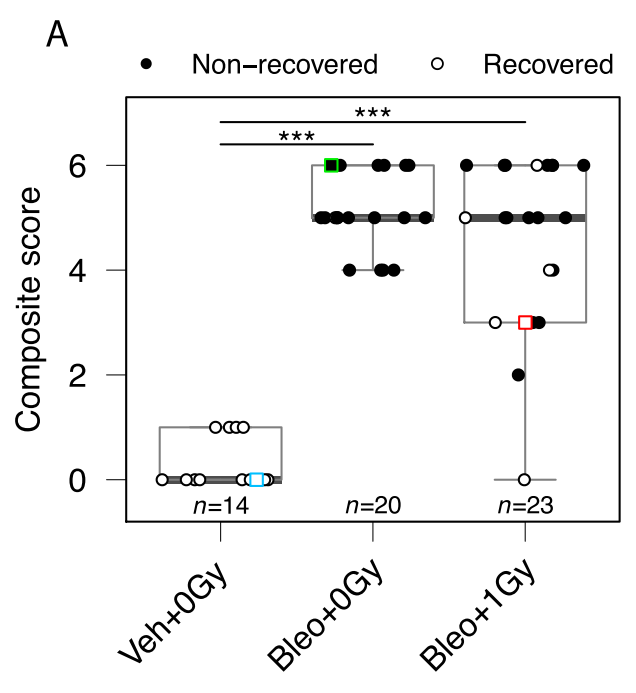

B
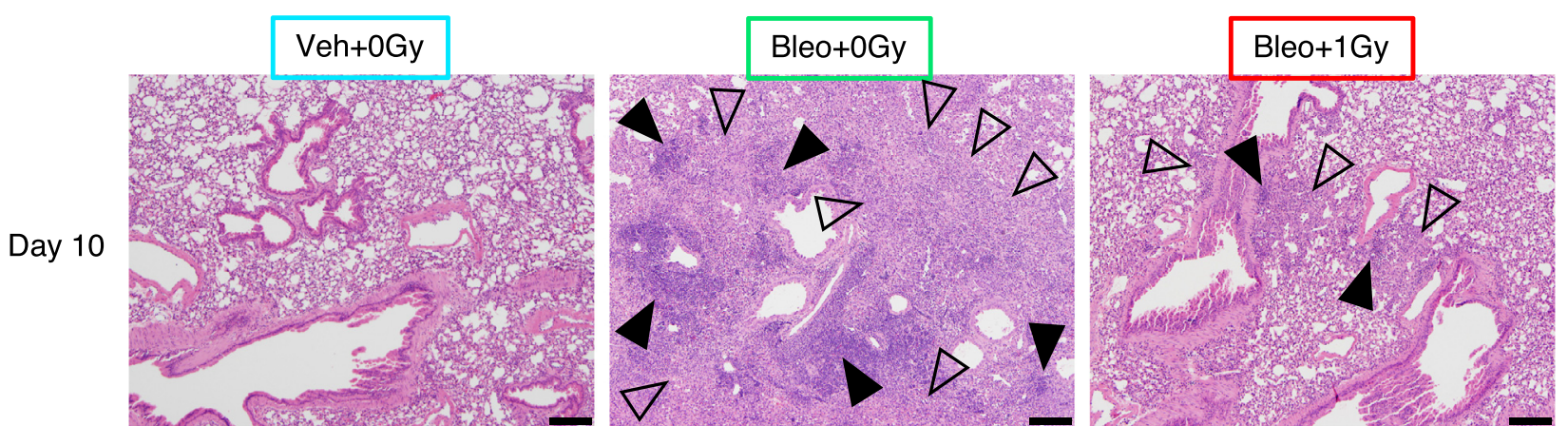

Fig. 3. Low-dose whole lung radiation therapy (LDLR) reduces severity of lung pathology in a subset of bleomycin-treated mice. (A) Histology composite scores of pulmonary macrophage infiltrates and lymphocyte aggregates at day 10 . Square symbols indicate images presented in (B). Mice whose bodyweight returned to $98 \%$ of baseline were classified as recovered. Groups compared by Kruskal-Wallis and post hoc Wilcoxon pairwise test, $* * * P<.001$. (B) Examples of pulmonary histology at day 10. Macrophage infiltrates are annotated with open arrows and lymphocyte aggregates with filled arrows. Images color-coded for cross-referencing with square symbols in (A). Hematoxylin and eosin stain, scale bars: $200 \mathrm{~mm}$.

response before irradiation (day 3), as indicated by bodyweight AUC, and response to LDLR. Of the mice receiving $1.0 \mathrm{~Gy}$, those that went on to recover had significantly higher AUC values at day 3 than those that did not recover (Fig. 2E). This analysis also confirmed that there was no significant difference in mean pre-LDLR AUC between irradiated and sham-irradiated mice and that mice experiencing a severe initial response to bleomycin (low day 3 AUC) were more likely to go on to experience severe weight loss (humane endpoint), regardless of further treatment.

Histologic assessment of mice on day 10 for macrophage infiltrates and lymphocyte aggregates demonstrated significant increases in immune infiltration of the lungs of bleomycin-treated mice compared with vehicle-treated mice (Fig. 3A,B). Although no statistically significant difference in composite score was detected between irradiated and sham-irradiated groups, a subset of irradiated mice, composed predominantly of mice whose bodyweight had recovered after treatment (open symbols in Fig. 3A), exhibited lower levels of inflammatory cells. In keeping with this observation, there was a significant negative correlation between histologic composite score and relative bodyweight AUC across all mice exposed to bleomycin ( $r=-$ $0.42, P=.0048$; Fig. E4). Early fibrotic changes were observed (Fig. E5) but were deemed not substantial enough to be quantified with existing scoring systems for fibrosis, which have been created and validated for later timepoints than those under investigation in this study.

Immunocytologic assessment of mouse lungs on day 10 showed that although the bleomycin-induced increase in interstitial macrophages was significantly blunted by lung irradiation (Fig. 4A), the associated reduction in alveolar macrophages was not affected (Fig. 4B). Bleomycin-associated increases in CD103+ dendritic cells (DCs) and neutrophil-DC hybrids ${ }^{34}$ were also significantly attenuated in mice exposed to 1.0 Gy LDLR (Fig. 4C,D). Representative FACS plots are shown in Figure E6. In addition to changes in cell number, bleomycin inhalation was associated with increased expression of the costimulatory molecule CD86 
A

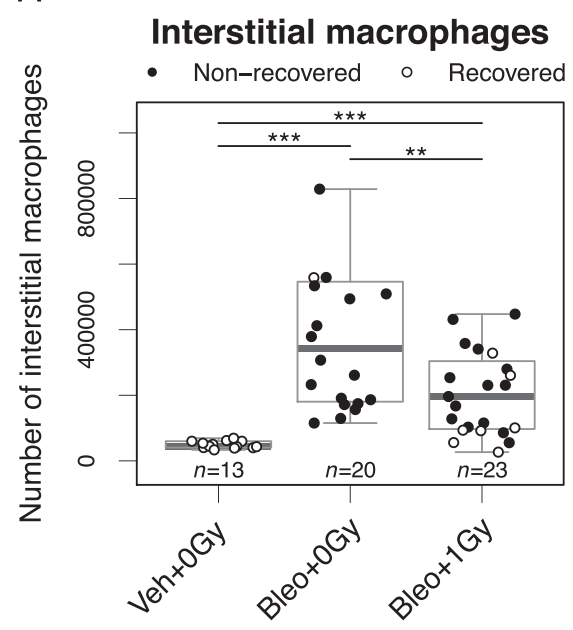

C

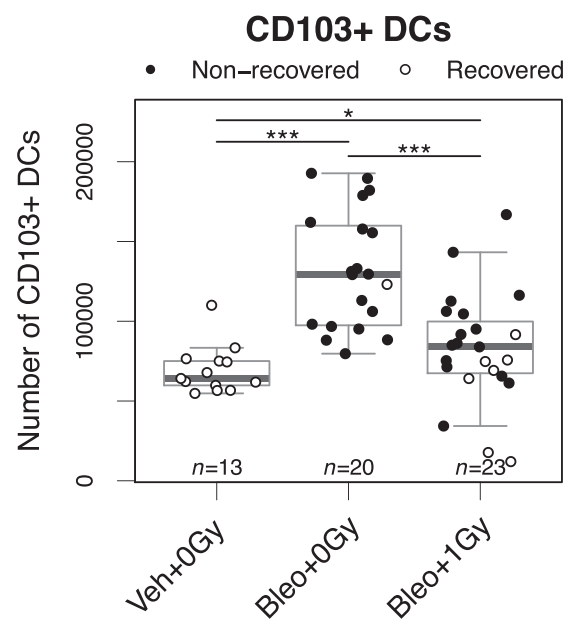

\section{B}

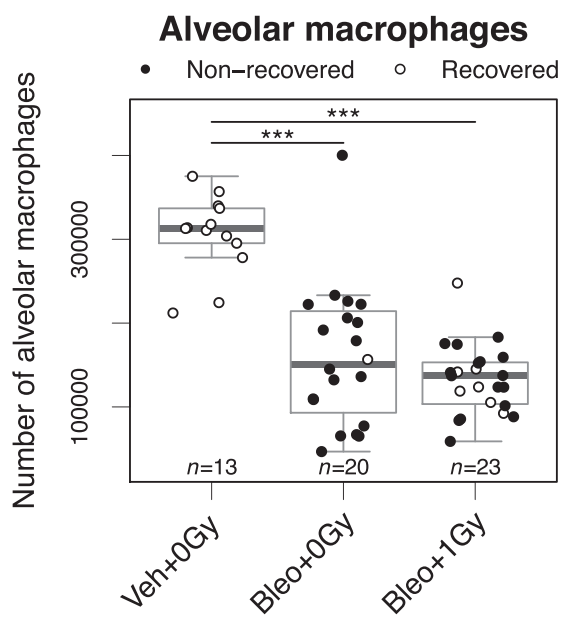

D

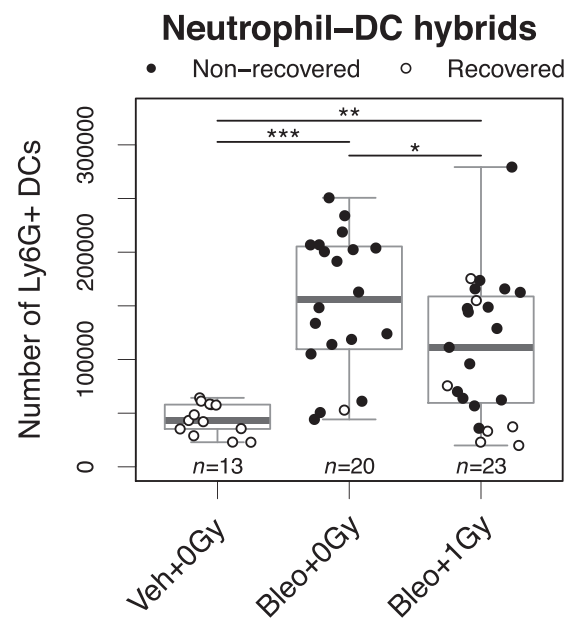

Fig. 4. Low-dose whole lung radiation therapy (LDLR). LDLR suppresses bleomycin-induced changes in immune cell numbers in mouse lung. Flow cytometric analysis of interstitial macrophages (A), alveolar macrophages (B), CD103+ dendritic cells (C), and Ly6G+ neutrophil-dendritic cell hybrids (D) in mouse lung at day 10 after bleomycin treatment. Mice whose bodyweight returned to $98 \%$ of baseline were classified as recovered. Groups compared by Kruskal-Wallis and post hoc Wilcoxon pairwise test, $* P<.05, * * P<.01, * * * P<.001$.

on alveolar macrophages and on neutrophil-DC hybrids but reduced expression on CD103+ dendritic cells and interstitial macrophages (Fig. E7). Importantly, the reduction in expression of CD86 induced by bleomycin in interstitial macrophages was significantly attenuated by LDLR at 1.0 Gy. The numbers of lymphocytes in lung tissue were not significantly affected by bleomycin treatment (Fig. E8).

To enable longitudinal assessment of lung infiltration, mice underwent CT imaging of the thorax on day 3 (preirradiation) and day 10 . As previously reported, bleomycinrelated changes were significantly more pronounced in the left lung (Fig. 5A,B); this is thought to be due to morphologic differences between left and right main bronchi. ${ }^{35}$ Left and right lung data sets were therefore analyzed separately. Consistent with evolving acute lung injury, aerated lung volume decreased between days 3 and 10 in sham-irradiated mice (both lungs) and in the left lungs of irradiated mice (Fig. 5C). In contrast, no statistically significant deterioration was observed in the right lungs of irradiated subjects (Fig. 5C, right panel). Furthermore, the mean decrease in right-lung aerated volume was significantly less in irradiated mice than in controls $(-3.8 \%$ and $-11.9 \%$ respectively, Fig. 5D). Indeed, 36\% $(\mathrm{n}=22)$ of irradiated mice showed an improvement (change $>0 \%$ ) in right lung aeration at day 10 , compared with only $5 \%(n=19)$ of controls. No effect of irradiation was observed in the left lungs. These observations are in keeping with the concept that LDLR has antiinflammatory efficacy in the context of moderate pneumonitis, as seen in the right lungs, but not the left lungs, which exhibited more severe changes. 


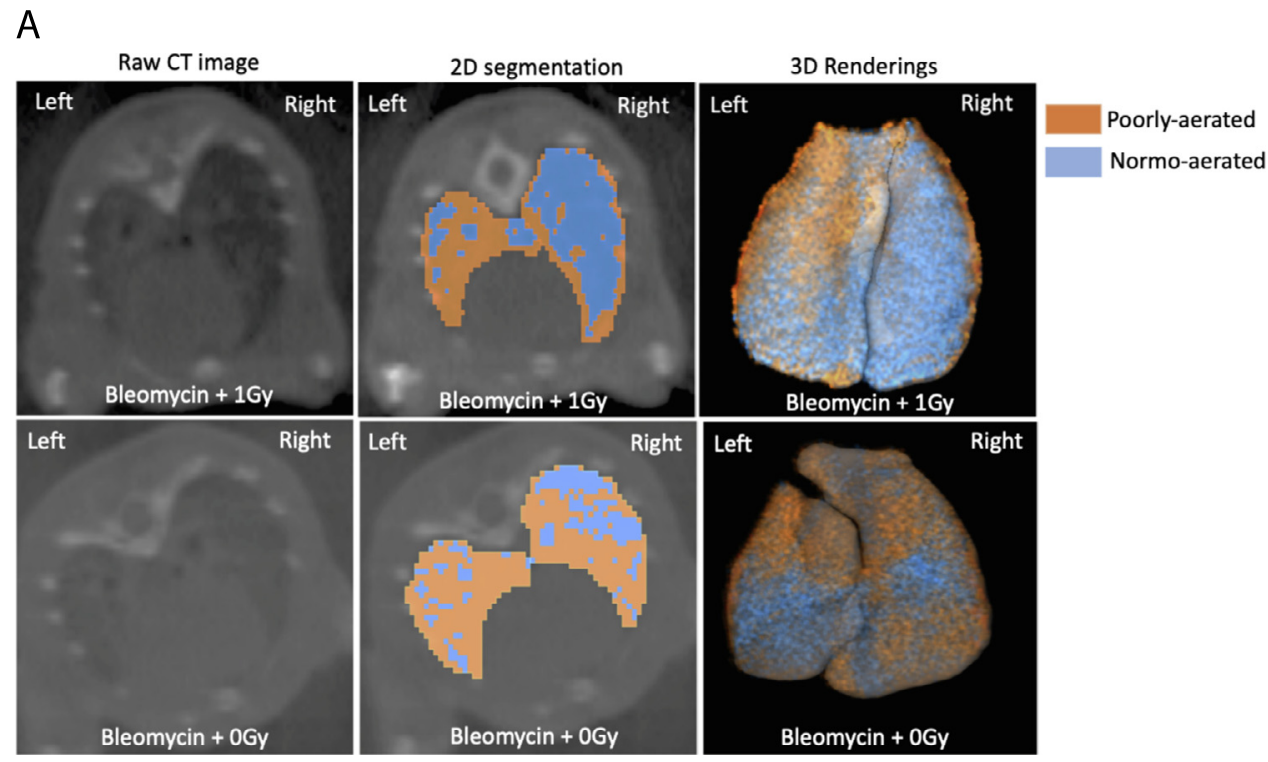

\section{B}

\section{Bleomycin-treated, day 3}

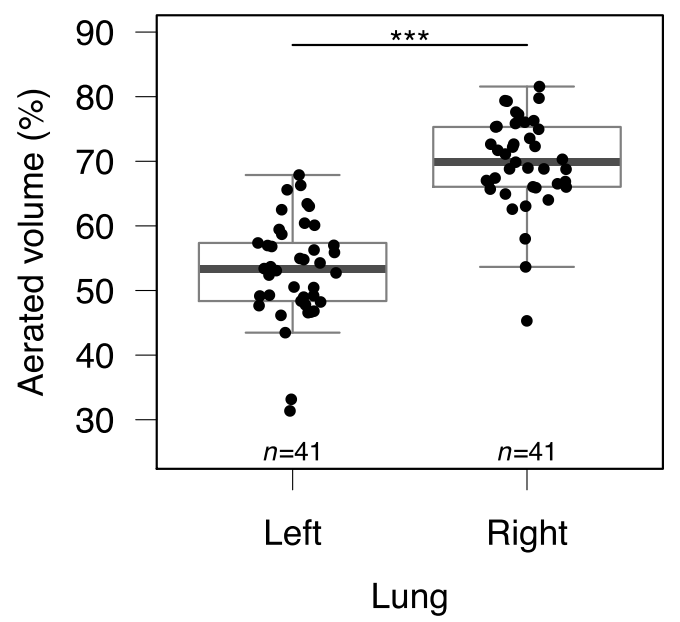

Fig. 5. Low-dose whole lung radiation therapy (LDLR) protects against bleomycin-induced radiologic changes in the right mouse lung. (A) The aerated volume of mouse lung was calculated from reconstructed computed tomography (CT) images. (B) Aerated volume percentage of each lung in all bleomycin-treated mice at day 3, before LDLR. Groups compared by $t$ test. (C) Percentage aerated volume of each lung on day 3 and day 10. The presence of a consistent trend between time points was assessed by paired $t$ test. (D) Change in aerated volume percentage between day 10 and day 3 (day $10-$ day 3 ) for each lung. Groups compared by $t$ test. $* P<.05,{ }^{* * *} P<.001$.

Finally, we evaluated 2 additional LDLR doses ( 0.5 and $1.5 \mathrm{~Gy})$ that are also being tested in clinical trials. Neither dose was associated with an improvement in outcome compared with sham irradiation, either in terms of mean bodyweight, likelihood of recovery, or survival probability (Fig. 6A, Fig. E9). In keeping with this, these doses did not dampen the bleomycin- associated lung infiltration of immune cell subsets that were previously shown to respond to $1.0 \mathrm{~Gy}$ (Fig. 6B). In fact, treatment with $1.5 \mathrm{~Gy}$ modestly but significantly exacerbated the bleomycin-induced reduction in lung alveolar macrophages. In contrast to 1.0 Gy, lung irradiation with 0.5 or 1.5 Gy failed to reduce deterioration of lung capacity as measured by CT imaging (Fig. 6C).

\section{Discussion}

Although a substantial body of clinical and preclinical data describes the immunomodulatory effects of low doses of 
C

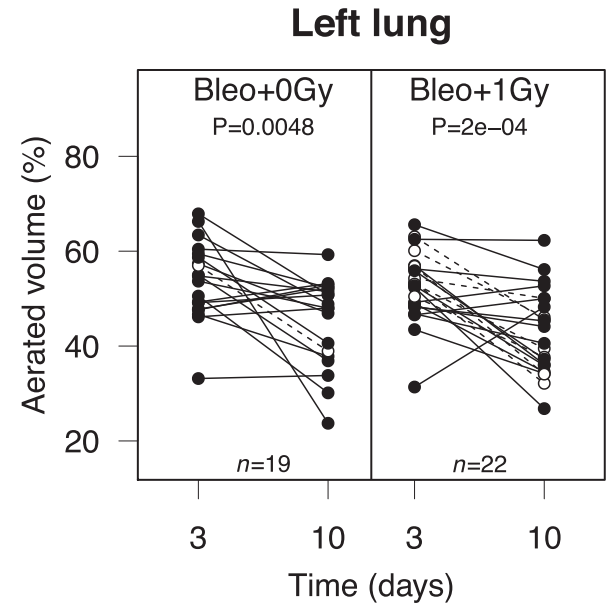

D

\section{Left lung}

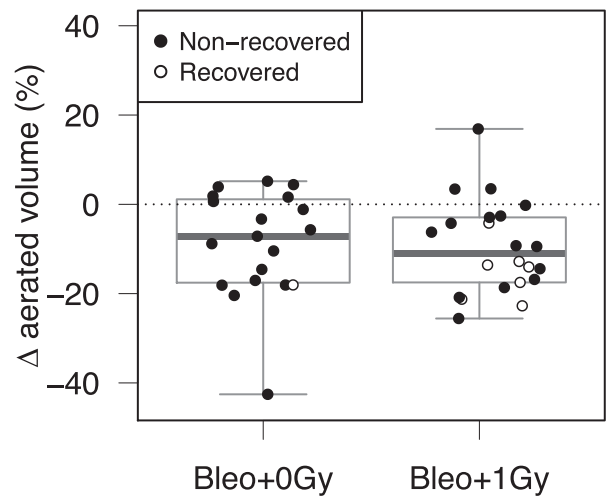

Right lung

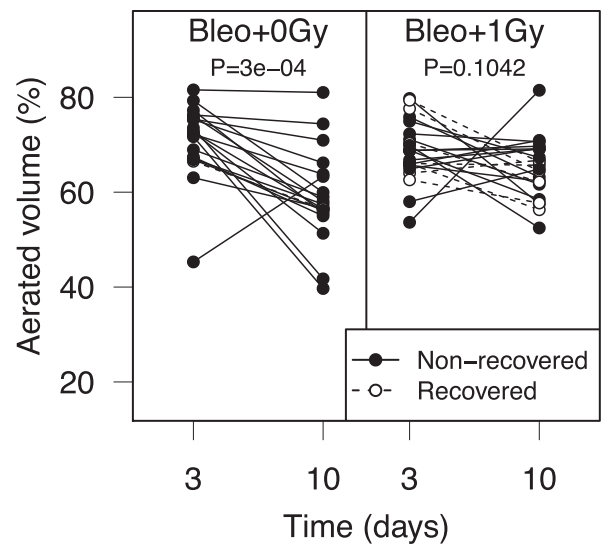

Right lung

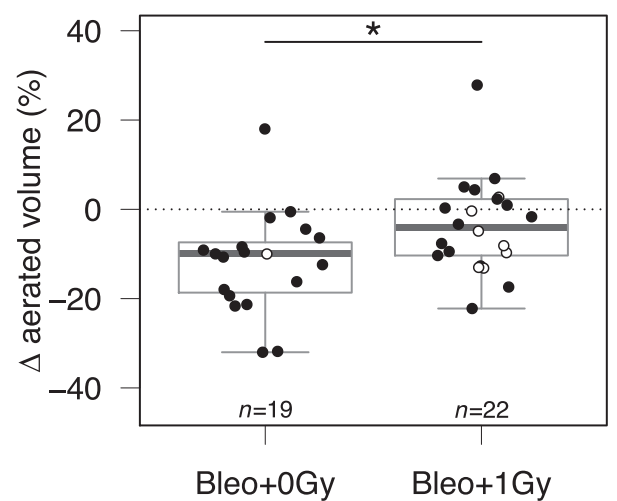

Fig. 5. Continued.
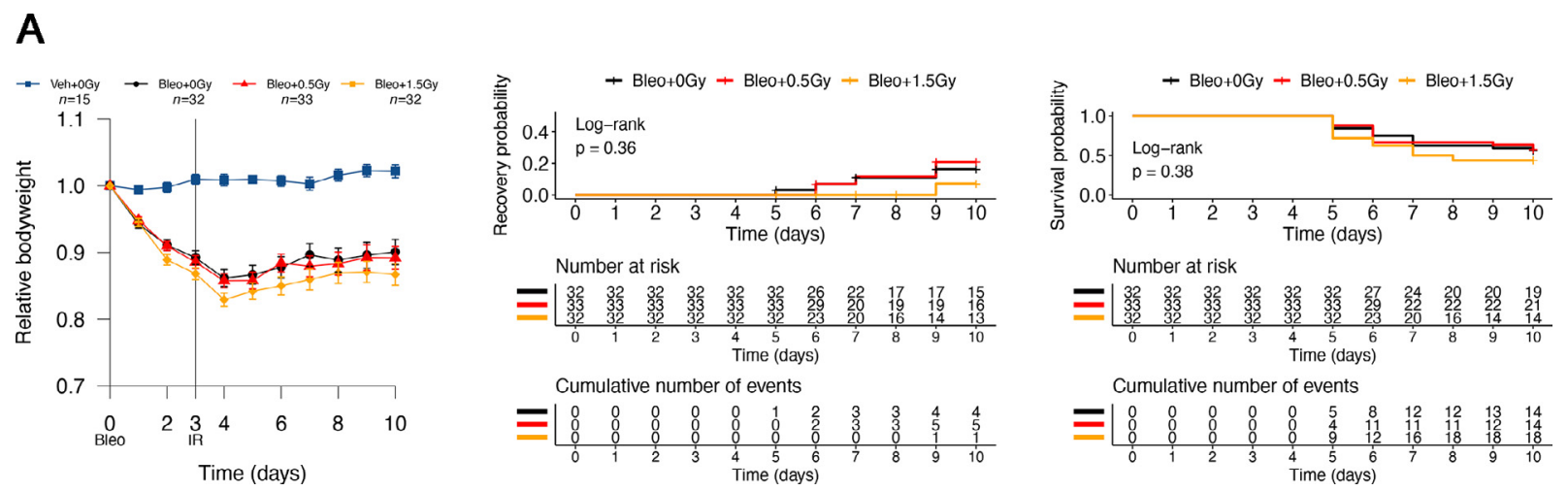

Fig. 6. Low-dose whole lung radiation therapy (LDLR) using 0.5 Gy or 1.5 Gy failed to improve outcomes of bleomycintreated mice. (A) Relative bodyweight (mean \pm standard error of the mean [SEM]) of bleomycin-treated mice after LDLR ( 0.5 or $1.5 \mathrm{~Gy}$ ) on day 3 (left panel). Kaplan-Meier analysis of recovery to $98 \%$ of baseline bodyweight (middle panel) and overall survival (right panel). Groups compared by log-rank test. (B) Flow cytometric analysis of immune cells in mouse lung at day 10. Groups compared by Kruskal-Wallis and post hoc Wilcoxon pairwise test. (C) Change in aerated lung volume percentage between day 10 and day 3, determined from serial computed tomography (CT) imaging (day $10-$ day 3 ) for each lung. Groups compared by 1 -way analysis of variance (ANOVA). $* P<.05, * * P<.01, * * * P<.001$. 
B
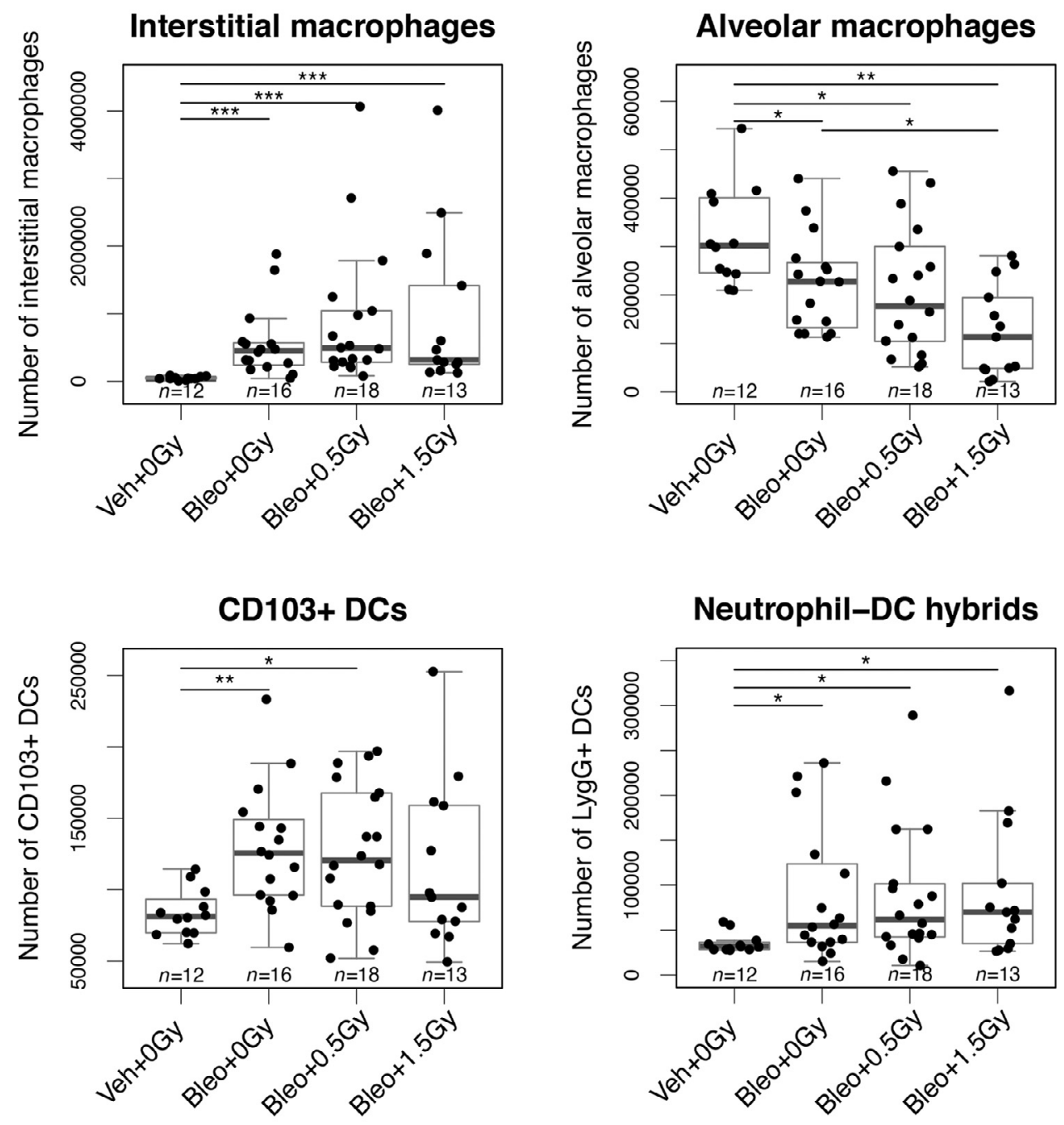

C
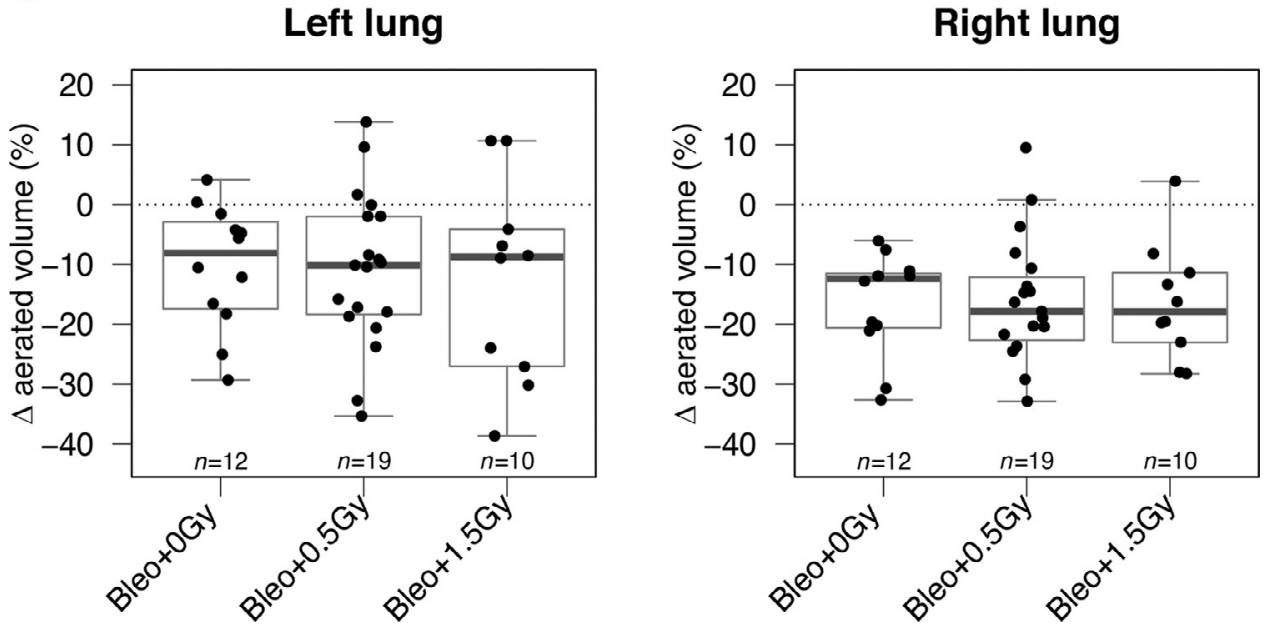

Fig. 6. Continued. 
radiation, none of the previously published work has studied pneumonitis. In the absence of a validated small animal model of COVID-19 lung disease ${ }^{36}$ and the urgent need for relevant preclinical data, we used the well-characterized bleomycin model to undertake pragmatic studies that we hope will provide useful data for clinicians developing early phase studies of LDLR in patients with COVID-19. The bleomycin model was selected because it exhibits many of the pathophysiologic changes associated with COVID-19 lung disease and because recent single cell sequencing studies support the existence of shared immunologic mechanisms. ${ }^{22-24}$ However, we recognize its limitations: Immune responses to bleomycin and SARS-CoV-2 are not identical, neither within the lungs nor systemically. Furthermore, our experiments were conducted exclusively in female mice aged 11 to 13 weeks. There is some evidence that young mice are less responsive to bleomycin than older mice, ${ }^{37}$ and it is possible that male mice would respond differently to bleomycin, LDLR, or both. Furthermore, it is well established that the risk of severe COVID-19 lung disease is much greater in older patients ${ }^{38}$ and that males are at higher risk of poor outcomes. ${ }^{39}$ Interactions with clinical comorbidities have also been described.

Having identified bodyweight as a clinically relevant primary endpoint that correlates with the severity of bleomycin-induced pneumonitis and the associated systemic inflammatory response, ${ }^{40}$ we observed wide variation between mice in terms of rapidity and severity of weight loss and subsequent recovery. Despite the challenges posed by this variability, our findings support the hypothesis that LDLR, delivered at a time when early histologic and immunologic features of lung inflammation are apparent, increases the likelihood of recovery in a subset of mice (approximately 25\%). These bodyweight data are supported by histologic, radiologic, and immunologic observations, which show that LDLR reduces the severity of bleomycininduced lung changes in a proportion of mice. Subsequent analyses indicated that mice with moderate pneumonitis (measured by lower rates of weight loss) were more likely to respond to LDLR than those with severe pneumonitis. These findings were corroborated by imaging studies that showed that LDLR reduced or even reversed the trajectory of bleomycin-induced changes in the right lungs, which were typically less severe than those in the left lungs, but had no effect on left lung imaging parameters. These observations are in keeping with recently published clinical data showing no benefit of LDLR in patients with severe COVID-19 pneumonia. ${ }^{14}$ Of note, histologic improvement was also observed in a subset of irradiated mice, even though this assessment was performed exclusively on left lung tissue, which was generally more severely affected than right lung tissue.

Because the various clinical studies underway are evaluating a range of lung radiation doses from 0.35 to $1.5 \mathrm{~Gy}$, we tested 3 different doses $(0.5,1.0$, and $1.5 \mathrm{~Gy})$ in an attempt to inform clinical decisions in this area. Of these, only 1.0 Gy demonstrated signs of efficacy, whereas $1.5 \mathrm{~Gy}$ showed some evidence of a detrimental effect. Cytologic analyses suggest that the efficacy of 1.0 Gy likely is associated with the fact that this was the only dose to significantly reduce immune infiltration of the lung. Our observations are largely consistent with the data recently published by Meziani et $\mathrm{al}^{11}$ in lipopolysaccharide and H1N1 influenza models of lung injury, although responses to 0.5 Gy were also observed in some of the histologic and cytologic readouts reported in that study.

Considering possible mechanisms, our cytologic studies showed that inhaled bleomycin caused an acute loss of alveolar macrophages and concomitant accumulation of myeloid cell populations in the lung. Similarly, in influenza virus infection, numbers of lung DCs increase as a consequence of more precursor cells migrating to the lung. ${ }^{41}$ Although LDLR was unable to prevent loss of alveolar macrophages, it did reduce accumulation of key DC, macrophage, and neutrophil populations (Fig. 4). A plausible explanation is that LDLR suppresses the signals that attract precursor DCs and/or inhibits their differentiation into CD103+ DCs. Infection or lung injury can lead to an accumulation of lung macrophages through either recruitment ${ }^{42}$ or local proliferation in a Th2-helper environment. ${ }^{43}$ Bleomycin treatment has been shown to increase production of the chemokine CCL2 by lung cells ${ }^{44,45}$; because migration of monocytes into inflamed lungs is dependent on CCL2/ CCR2 signalling, ${ }^{44,45}$ it is reasonable to propose that LDLR might act by reducing CCL2 and/or other signals that attract monocytes into the damaged lung.

Chemokines such as MIP-2 and CXCL5 are released in the first few days after acute lung injury, and, together with other factors including extracellular adenosine triphosphate, may play a role in initiating and sustaining accumulation of neutrophils within the lungs after bleomycin inhalation. ${ }^{46}$ Although we saw no increase in classical neutrophils in bleomycin-exposed lungs, we did observe accumulation of a hybrid population that expressed markers of both neutrophils (Ly6G) and DCs (CD11c, MHCII). These hybrid cells are thought to differentiate from neutrophil precursors, retaining their phagocytic function while gaining the ability to present antigen to CD4 T cells. ${ }^{34}$ Inflammation induced by thioglycolate or bacterial or fungal infection leads to an increase in this hybrid population in mouse models of tissue inflammation, and these cells have also been found in human tumors. ${ }^{47-49}$ Our data extend these observations to show that bleomycin also drives accumulation and differentiation of these cells, an effect that we showed to be significantly blunted by LDLR.

\section{Conclusions}

Our data provide preclinical evidence of efficacy of LDLR in a subset of mice with moderate lung injury induced by bleomycin, identify $1.0 \mathrm{~Gy}$ as the most effective radiation 
dose tested, and reveal plausible immunologic mechanisms. More comprehensive studies in additional models of pneumonitis and over longer observation periods are warranted to inform ongoing and future studies in patients with COVID-19.

\section{References}

1. Johns Hopkins University Coronavirus Resource Center. Available at: https://coronavirus.jhu.edu/map.html. Accessed February 24, 2021.

2. Guan WJ, Ni ZY, Hu Y, et al. Clinical characteristics of coronavirus disease 2019 in China. New Engl J Med 2020;382:1708-1720.

3. Tay MZ, Poh CM, Renia L, MacAry PA, Ng LFP. The trinity of COVID-19: Immunity, inflammation and intervention. Nat Rev Iттиnol 2020;20:363-374.

4. Mehta P, McAuley DF, Brown M, et al. COVID-19: Consider cytokine storm syndromes and immunosuppression. Lancet 2020;395: 1033-1034.

5. St John AL, Rathore APS. Early insights into immune responses during COVID-19. J Immunol 2020;205:555-564.

6. Laing AG, Lorenc A, Del Molino Del Barrio I, et al. A dynamic COVID-19 immune signature includes associations with poor prognosis. Nat Med 2020;26:1623-1635.

7. Group RC, Horby P, Lim WS, et al. Dexamethasone in hospitalized patients with covid-19. N Engl J Med 2021;384:693-704.

8. Lopes MI, Bonjorno LP, Giannini MC, et al. Beneficial effects of colchicine for moderate to severe COVID-19: A randomised, doubleblinded, placebo-controlled clinical trial. RMD Open 2021;7:e001455.

9. Calabrese EJ, Dhawan G. How radiotherapy was historically used to treat pneumonia: Could it be useful today? Yale J Biol Med 2013;86:555-570

10. Rodel F, Keilholz L, Herrmann M, Sauer R, Hildebrandt G. Radiobiological mechanisms in inflammatory diseases of low-dose radiation therapy. Int J Radiat Biol 2007;83:357-366.

11. Meziani L, Robert C, Classe M, et al. Low doses of radiation increase the immunosuppressive profile of lung macrophages during viral infection and pneumonia. Int $J$ Radiat Oncol Biol Phys 2021:110:1283-1294

12. Hess CB, Buchwald ZS, Stokes W, et al. Low-dose whole-lung radiation for COVID-19 pneumonia: Planned day 7 interim analysis of a registered clinical trial. Cancer 2020;126:5109-5113.

13. Sanmamed N, Alcantara P, Cerezo E, et al. Low-dose radiation therapy in the management of coronavirus disease 2019 (COVID-19) pneumonia (LOWRAD- Cov19): Preliminary report. Int J Radiat Oncol Biol Phys 2021;109:880-885.

14. Papachristofilou A, Finazzi T, Blum A, et al. Low dose radiation therapy for severe COVID-19 pneumonia: A randomized double-blind study. Int J Radiat Oncol Biol Phys 2021;110:1274-1282.

15. Kirsch DG, Diehn M, Cucinotta FA, Weichselbaum R. Lack of supporting data make the risks of a clinical trial of radiation therapy as a treatment for COVID-19 pneumonia unacceptable. Radiother Oncol 2020;147:217-220.

16. Garcia-Hernandez T, Romero-Exposito M, Sanchez-Nieto B. Low dose radiation therapy for COVID-19: Effective dose and estimation of cancer risk. Radiother Oncol 2020;153:289-295.

17. Cuttler JM, Bevelacqua JJ, Mortazavi SMJ. Unethical not to investigate radiotherapy for COVID-19. Dose Response 2020;18: 1559325820950104

18. Hanna CR, Robb KA, Blyth KG, Jones RJ, Chalmers AJ. Clinician attitudes to using low-dose radiation therapy to treat COVID-19 lung disease. Int J Radiat Oncol Biol Phys 2021;109:886-890.

19. Prasanna PG, Woloschak GE, DiCarlo AL, et al. Low-dose radiation therapy (LDRT) for COVID-19: Benefits or risks? Radiat Res 2020;194:452-464.
20. Matute-Bello G, Frevert CW, Martin TR. Animal models of acute lung injury. Am J Physiol Lung Cell Mol Physiol 2008;295:L379L399.

21. Bordag N, Biasin V, Schnoegl D, et al. Machine learning analysis of the bleomycin mouse model reveals the compartmental and temporal inflammatory pulmonary fingerprint. iScience 2020;23: 101819.

22. Aran D, Looney AP, Liu L, et al. Reference-based analysis of lung single-cell sequencing reveals a transitional profibrotic macrophage. Nat Immunol 2019;20:163-172.

23. Liao M, Liu Y, Yuan J, et al. Single-cell landscape of bronchoalveolar immune cells in patients with COVID-19. Nat Med 2020;26:842-844.

24. Wen W, Su W, Tang H, et al. Immune cell profiling of COVID-19 patients in the recovery stage by single-cell sequencing. Cell Discov 2020;6:31.

25. Urawa M, Kobayashi T, D'Alessandro-Gabazza CN, et al. Protein S is protective in pulmonary fibrosis. J Thromb Haemost 2016;14:15881599.

26. Lemke G, Silverman GJ. Blood clots and TAM receptor signalling in COVID-19 pathogenesis. Nat Rev Immunol 2020;20:395-396.

27. Fedorov A, Beichel R, Kalpathy-Cramer J, et al. 3D Slicer as an image computing platform for the Quantitative Imaging Network. Magn Reson Imaging 2012;30:1323-1341.

28. Harvard University Chest Imaging Platform. Available at: https://ches timagingplatform.org/. Accessed February 1, 2021.

29. Misharin AV, Morales-Nebreda L, Mutlu GM, Budinger GR, Perlman H. Flow cytometric analysis of macrophages and dendritic cell subsets in the mouse lung. Am J Respir Cell Mol Biol 2013;49:503-510.

30. R Core Team. A language and environment for statistical computing. R Foundation for Statistical Computing. Available at: https://www.Rproject.org/. Accessed February 1, 2021.

31. Ekstrøm CT. MESS: Miscellaneous Esoteric Statistical Scripts. R package version 0.5.6. 2019.

32. Therneau $T$. A package for survival analysis in $R_{-}$. R package version 3.1-11. Available at: https://CRAN.R-project.org/package=survival. Accessed February 1, 2021.

33. Kassambara AKK, Biecek P. Drawing survival curves using 'ggplot 2 '. R package version 0.4.6. Available at: https://CRAN.R-project.org/ package $=$ survminer. Accessed September 1, 2021.

34. Matsushima H, Geng S, Lu R, et al. Neutrophil differentiation into a unique hybrid population exhibiting dual phenotype and functionality of neutrophils and dendritic cells. Blood 2013;121:1677-1689.

35. Thiesse J, Namati E, Sieren JC, et al. Lung structure phenotype variation in inbred mouse strains revealed through in vivo micro-CT imaging. J Appl Physiol 2010;109:1960-1968.

36. Lakdawala SS, Menachery VD. The search for a COVID-19 animal model. Science 2020;368:942-943.

37. Redente EF, Jacobsen KM, Solomon JJ, et al. Age and sex dimorphisms contribute to the severity of bleomycin-induced lung injury and fibrosis. Am J Physiol Lung Cell Mol Physiol 2011;301:L510L518.

38. Wang D, Hu B, Hu C, et al. Clinical characteristics of 138 hospitalized patients with 2019 novel coronavirus-infected pneumonia in Wuhan, China. JAMA 2020;323:1061-1069.

39. Peckham H, de Gruijter NM, Raine C, et al. Male sex identified by global COVID-19 meta-analysis as a risk factor for death and ITU admission. Nat Commun 2020;11:6317.

40. Cowley PM, Roberts CR, Baker AJ. Monitoring the health status of mice with bleomycin-induced lung injury by using body condition scoring. Comp Med 2019;69:95-102.

41. Cabeza-Cabrerizo M, van Blijswijk J, Wienert S, et al. Tissue clonality of dendritic cell subsets and emergency DCpoiesis revealed by multicolor fate mapping of DC progenitors. Sci Immunol 2019;4.

42. Shi C, Pamer EG. Monocyte recruitment during infection and inflammation. Nat Rev Immunol 2011;11:762-774. 
43. Jenkins SJ, Ruckerl D, Cook PC, et al. Local macrophage proliferation, rather than recruitment from the blood, is a signature of $\mathrm{TH} 2$ inflammation. Science 2011;332:1284-1288.

44. Liang J, Jung Y, Tighe RM, et al. A macrophage subpopulation recruited by CC chemokine ligand-2 clears apoptotic cells in noninfectious lung injury. Am J Physiol Lung Cell Mol Physiol 2012;302: L933-L940.

45. Reader BF, Sethuraman S, Hay BR, et al. IRAK-M regulates monocyte trafficking to the lungs in response to bleomycin challenge. $J$ Immunol 2020;204:2661-2670.

46. Shah D, Romero F, Stafstrom W, Duong M, Summer R. Extracellular ATP mediates the late phase of neutrophil recruitment to the lung in murine models of acute lung injury. Am J Physiol Lung Cell Mol Physiol 2014;306:L152-L161.

47. Geng S, Matsushima H, Okamoto T, et al. Emergence, origin, and function of neutrophil-dendritic cell hybrids in experimentally induced inflammatory lesions in mice. Blood 2013;121:1690-1700.

48. Fites JS, Gui M, Kernien JF, et al. An unappreciated role for neutrophil-DC hybrids in immunity to invasive fungal infections. PLoS Pathog 2018;14:e1007073.

49. Singhal S, Bhojnagarwala PS, O'Brien S, et al. Origin and role of a subset of tumor-associated neutrophils with antigen-presenting cell features in early-stage human lung cancer. Cancer Cell 2016;30: $120-135$. 